\title{
Influência da postura na pressão intra-ocular e nos defeitos de campo visual no glaucoma primário de ângulo aberto e glaucoma de pressão normal
}

\section{Postural influence on intraocular pressure and visual field defects in open angle glaucoma and normal tension glaucoma}

Sérgio Henrique Sampaio Meirelles', Cristina Rodrigues Mathias², Gilberto Brandão ${ }^{3}$, Ana Carolina de Arantes Frota $^{4}$, Riuitiro Yamane ${ }^{5}$

\begin{tabular}{|l|c||}
\hline \multicolumn{1}{|c|}{ RESUMO } \\
Objetivo:Avaliar a variação da pressão intra-ocular (PIO) na mudança de posição em \\
pacientes com glaucoma primário de ângulo aberto (GPAA), glaucoma de pressão nor- \\
mal (GPN) e olhos normais (ON), e correlacionar esta variação à gravidade do defeito \\
de campo visual. Métodos: Foram avaliados 32 olhos de 32 pacientes com GPAA, 30 \\
olhos de 30 pacientes com GPN e 20 olhos de 20 ON.A PIO foi aferida às 6h da manhã na \\
posição supina e 5min após com o paciente sentado. Foi avaliada a correlação entre \\
variação da pressão decorrente da mudança de posição e os defeitos de campo visual. \\
Foram utilizados os testes estatísticos não paramétricos de Kruskal-Wallis, Wilcoxon, \\
Mann-Whitney e o teste de correlação linear de Spearman. Foi considerado significativo \\
valor de p menor que 0,05.Resultados: Foi encontrada diferença significativa entre as \\
pressões intra-oculares na posição supina e assentada nos três grupos (p<0,001). Não \\
houve diferença significativa na variação da PIO com a mudança de posição entre os três \\
grupos (p=0,17). Não houve correlação significativa entre a variação da PIO nas duas \\
posições e o índice MD do campo visual nos grupos GPAA (p=0,81) e GPN (p=0,89). \\
Conclusão:Os resultados sugerem que existe aumento significativo da PIO com a mudan- \\
ça da posição assentada para supina em pacientes com GPAA, GPN e ON, não havendo, \\
porém, diferença significativa desta variação entre os três grupos. Não foi encontrada \\
correlação significativa entre a variação da PIO e a severidade do campo visual.
\end{tabular}

Descritores: Glaucoma de ângulo aberto/diagnóstico; Pressão intra-ocular;Técnicas de diagnóstico oftalmológico;Tonometria ocular/métodos; Estudos de casos e controle

\footnotetext{
'Doutor pela Universidade Federal do Rio de Janeiro, Professor Assistente da Universidade Gama Filho, chefe do setor de glaucoma do Hospital Municipal da Piedade - Rio de Janeiro (RJ), Brasil.

${ }^{2}$ Médica do Setor de Glaucoma do Hospital Municipal da Piedade - Rio de Janeiro (RJ), Brasil.

${ }^{3}$ Médico do Setor de Glaucoma do Hospital Municipal da Piedade - Rio de Janeiro (RJ), Brasil.

${ }^{4}$ Médica do Setor de Glaucoma do Hospital Municipal da Piedade - Rio de Janeiro (RJ), Brasil.

${ }^{5}$ Livre Docente e Professor Titular da Universidade do Estado do Rio de Janeiro - UERJ - Rio de Janeiro (RJ), Brasil.

Instituição: Hospital Municipal da Piedade - Universidade Gama Filho - Rio de Janeiro (RJ), Brasil.

Recebido para publicação em: 14/11/2007 - Aceito para publicação em 16/2/2008
} 


\section{INTRODUÇÃO}

D iversos estudos têm demonstrado a variaçãoda pressão intra-ocular (PIO) decorrente damudança de posição. Pacientes em posição supina apresentam valores pressóricos mais elevados em relação às medidas obtidas com o paciente senta$\mathrm{do}^{12 .}$. Têm sido verificados picos de PIO às seis horas da manhã na posição supina, em pacientes submetidos à curva de PIO de 24 horas $^{3}$.

A elevação da PIO ao amanhecer, na posição supina, teria relação com mecanismos circadianos e com o aumento da pressão venosa episcleral ${ }^{45}$. A variação da $\mathrm{PIO}$ entre as posturas assentada e supina em pacientes glaucomatosos, pode ser maior que em indivíduos normais.'Pacientes com glaucoma de pressão normal (GPN) podem, também, apresentar variações significativas da PIO com a mudança de posição supina para assentada ${ }^{6.8}$.

A variação da PIO que ocorre ao se mudar a postura, pode ter relação com a severidade do glaucoma. Um estudo encontrou maiores variações da PIO com a mudança de posição em olhos de pacientes portadores de glaucoma primário de ângulo aberto que apresentavam maiores defeitos de campo visual, comparados aos olhos contra-laterais. Foi encontrado também, em outro trabalho, correlação entre a progressão de defeitos de campo visual e o aumento da PIO provocada pela alteração da postura em pacientes portadores de $\mathrm{GPN}^{10}$. Este dado pode ser importante na avaliação da terapêutica de pacientes glaucomatosos. Assim, a avaliação da pressão intra-ocular em posição supina poderia auxiliar na detecção de picos pressóricos não evidenciados em tonometrias realizadas no consultório, ou mesmo em uma curva ambulatorial de pressão intra-ocular.

O objetivo deste trabalho é avaliar a variação da pressão intra-ocular com a mudança de posição em pacientes com glaucoma primário de ângulo aberto (GPAA), GPN e indivíduos com olhos normais (ON),e correlacionar esta variação com a gravidade de defeito do campo visual.

\section{Métodos}

Foram avaliados pacientes atendidos no Setor de Glaucoma do Hospital Municipal da Piedade, de dezembro de 2003 a dezembro de 2004. Todos os pacientes assinaram um termo de livre consentimento e foram submetidos a exame oftalmológico completo. O protocolo da pesquisa foi aprovado pela Comissão de Ética do Hospital Municipal da Piedade, onde foi realizado o tra- balho. Os pacientes foram divididos em três grupos: glaucoma primário de ângulo aberto, glaucoma de pressão normal e indivíduos normais.

Os pacientes com GPAA apresentavam pelo menos duas medidas da PIO maiores que $21 \mathrm{mmHg}$, com lesão do nervo óptico característica de glaucoma ${ }^{11}$ e defeitos correspondentes de campo visual ${ }^{12}$. Todos os pacientes apresentavam a gonioscopia, ângulo aberto sem anormalidades e não possuíam nenhuma outra doença ocular ou história de cirurgia ocular ou doença sistêmica ou neurológica que justificasse as alterações do nervo óptico e campo visual.

Os pacientes com glaucoma de pressão normal (GPN) apresentavam os mesmos critérios de inclusão do grupo GPAA, exceto que não apresentavam nenhuma medida da PIO maior que $21 \mathrm{mmHg}$ em uma curva diária de pressão de 24 horas, e com lesões do nervo óptico características de glaucoma ${ }^{11}$, e defeitos correspondentes de campo visual ${ }^{12}$.

Os indivíduos normais do grupo controle (ON) apresentavam medidas da pressão intra-ocular menores que $21 \mathrm{mmHg}$ em diversas medidas da $\mathrm{PIO}$, sem alterações do nervo óptico e do campo visual e com exame oftalmológico normal.

Nenhum dos pacientes apresentava qualquer outra patologia ocular, havia sido submetido à cirurgia intra-ocular ou estava em uso de qualquer medicação, antiglaucomatosa ou não, que pudesse interferir na PIO. O diagnóstico dos pacientes foi realizado durante o estudo, motivo pelo qual nenhum paciente estava em uso de medicação antiglaucomatosa.

Todos os pacientes realizaram exame oftalmológico completo com acuidade visual corrigida, biomicroscopia, tonometria de aplanação, biomicroscopia do disco óptico com lente de 78 dioptrias, gonioscopia com lente de dois espelhos, curva diária de pressão de 24 horas e exame de campo visual computadorizado com estratégia Fast-threshold 24-2 do aparelho Oculus Centerfield ${ }^{\oplus}$ (Dutenhofen, Alemanha) e estímulo branco em fundo branco. Todos os pacientes realizaram pelo menos dois exames de campo visual, reprodutíveis e confiáveis.

Para efeito do estudo foram considerados as medidas das 6 horas da curva diária de pressão com o paciente em posição supina e 5 minutos após em posição assentada. As duas medidas foram realizadas com o tonômetro de aplanação de Perkins. Todas as medições da PIO foram realizadas pelo mesmo examinador. Foi avaliado apenas o olho direito de cada paciente.

Foram utilizados os testes estatísticos não 
Tabela 1

Média e desvio padrão da idade dos três grupos

\begin{tabular}{lcc}
\hline & N & Idade (anos) \\
\hline GPAA & 32 & $58,8 \pm 13,6$ \\
GPN & 30 & $59,6 \pm 11,7$ \\
ON & 20 & $52,6 \pm 13,4$ \\
Valor de p & & 0,12 \\
\hline
\end{tabular}

Teste estatístico: teste de Kruskal-Wallis. $\mathrm{p}=0,05$

GPAA: glaucoma primário de ângulo aberto; GPN: glaucoma de pressão normal; ON: olhos normais.

Tabela 3

Pressão intra-ocular na posição supina entre os 3 grupos

\begin{tabular}{lcc}
\hline & N & PIO (mmHg) \\
\hline GPAA & 32 & $23,47 \pm 4,81$ \\
GPN & 30 & $18,3 \pm 2,2$ \\
ON & 20 & $15 \pm 2,57$ \\
\hline Valor de p & $<0,001$ \\
\hline Dunn & & Valor de $\mathrm{p}$ \\
\hline GPAA e GPN & $\mathrm{p}<0,001$ \\
GPAA e IN & $\mathrm{p}<0,001$ \\
GPN e IN & $\mathrm{p}<0,001$ \\
\hline
\end{tabular}

Teste estatístico: Teste de Kruskal-Wallis com teste de Dunn. $\mathrm{p}=0,05$.

GPA: glaucoma primário de ângulo aberto; GPN: glaucoma de pressão normal.

ON: olhos normais. PIO: pressão intra-ocular.

Tabela 5

Média e desvio padrão da variação da pressão intra-ocular entre posição supina e assentada entre os 3 grupos

\begin{tabular}{lcc}
\hline & N & PIO (mmHg) \\
\hline GPAA & 32 & $3,41 \pm 1,95$ \\
GPN & 30 & $2,77 \pm 1,61$ \\
IN & 20 & $2,45 \pm 1,57$ \\
Valor de p & & $\mathrm{p}=0,17$ \\
\hline
\end{tabular}

Teste estatístico: teste de Kruskal-Wallis. $\mathrm{p}=0,05$

GPAA: glaucoma primário de ângulo aberto; GPN: glaucoma de pressão normal; ON: olhos normais
Tabela 2

Média e desvio padrão da pressão intra-ocular em posição supina e assentada dos 3 grupos

\begin{tabular}{lcccc}
\hline & N & $\begin{array}{c}\text { PIO supina } \\
(\mathbf{m m H g})\end{array}$ & $\begin{array}{c}\text { Pio assentada } \\
(\mathbf{m m H g})\end{array}$ & Valor de p \\
\hline GPAA & 32 & $23,47 \pm 4,81$ & $20,06 \pm 4,29$ & $<0,001$ \\
GPN & 30 & $18,3 \pm 2,2$ & $15,53 \pm 2,13$ & $<0,001$ \\
ON & 20 & $15 \pm 2,57$ & $12,55 \pm 2,39$ & $<0,001$ \\
\hline
\end{tabular}

Teste estatístico: teste de Wilcoxon - teste $\mathrm{T}$ para amostras pareadas. $\mathrm{p}=0,05$

GPAA: glaucoma primário de ângulo aberto; GPN: glaucoma de pressão normal; ON: olhos normais.

Tabela 4

Pressão intra-ocular na posição assentada entre os 3 grupos

\begin{tabular}{lcc}
\hline & N & PIO (mmHg) \\
\hline GPAA & 32 & $20,06 \pm 4,29$ \\
GPN & 30 & $15,53 \pm 2,13$ \\
ON & 20 & $12,55 \pm 2,39$ \\
\hline Valor de p & $<0,001$ \\
\hline Dunn & Valor de $\mathrm{p}$ \\
\hline GPAA e GPN & $\mathrm{p}<0,001$ \\
GPAA e IN & $\mathrm{p}<0,001$ \\
GPN e IN & $\mathrm{p}<0,001$ \\
\hline
\end{tabular}

Teste estatístico: Teste de Kruskal-Wallis com teste de Dunn. $\mathrm{p}=0,05$.

GPA: glaucoma primário de ângulo aberto; GPN: glaucoma de pressão normal

ON: olhos normais. PIO: pressão intra-ocular.

Tabela 6

Correlação linear entre a variação da pressão intra-ocular e o índice "mean defect" (MD) da perimetria computadorizada

\begin{tabular}{lcc}
\hline & $\mathbf{r}_{\mathbf{s}}$ & Valor de p \\
\hline GPAA & $-0,04$ & 0,81 \\
GPN & 0,03 & 0,89 \\
\hline
\end{tabular}

Teste estatístico: teste de correlação linear de Spearman. p=0,05 GPA: glaucoma primário de ângulo aberto; GPN: glaucoma de pressão normal; PIO: pressão intra-ocular; r : Coeficiente de correlação de Spearman. 
Tabela 7

Média e desvio padrão da variação da pressão intra-ocular entre a posição supina e assentada do melhor e do pior olho de cada paciente de acordo com o índice MD, dos grupos GPAA e GPN

\begin{tabular}{lccc}
\hline & $\begin{array}{c}\text { Diferença PIO } \\
\text { melhor olho } \\
\text { (mmHg) }\end{array}$ & $\begin{array}{c}\text { Diferença PIO } \\
\text { pior olho } \\
\text { (mmHg) }\end{array}$ & Valor de p \\
\hline GPAA & $2,97 \pm 2,16$ & $3,59 \pm 2,2$ & 0,31 \\
GPN & $2,73 \pm 1,23$ & $2,73 \pm 1,87$ & 0,99 \\
\hline
\end{tabular}

Teste estatístico: teste de Mann-Whitney para amostras independentes. $\mathrm{p}=0,05$

GPA: glaucoma primário de ângulo aberto; GPN: glaucoma de pressão normal; PIO: pressão intra-ocular.

paramétricos de Kruskal-Wallis, Wilcoxon,Mann-Whitney e o teste de correlação linear de Spearman pelos programas BioEstat $3.0^{\oplus} \mathrm{e} \mathrm{Excel}^{\oplus}$. Foi considerado significativo valor de $\mathrm{p}$ menor que 0,05 , com poder das amostras de 0,90 para diferença entre as PIOs de $2 \mathrm{mmHg}$.

\section{Resultados}

A amostra foi constituída de 82 pacientes, sendo 32 portadores de GPAA, 30 pacientes com GPN e 20 indivíduos com olhos normais. O grupo I (GPAA) foi constituído de 32 pacientes com GPAA, sendo 23 do sexo feminino e 8 do sexo masculino, com idade média de 58,8 anos, variando de 36 a 81 anos. O grupo II (GPN) foi composto por 30 pacientes portadores de GPN, sendo 25 do sexo feminino e 5 do sexo masculino, com idade média de 59,6 anos, variando de 38 a 80 anos. O grupo III (ON) foi constituído de 20 indivíduos com olhos normais, sendo 12 do sexo feminino, 8 do sexo masculino, com idade média de 52,6 anos, variando de 36 a 82 anos. Não houve diferença estatisticamente significativa entre a idade dos pacientes dos três grupos (Tabela 1).

A tabela 2 mostra que houve diferença significativa entre as médias obtidas da PIO na posição supina e na posição assentada nos três grupos, sendo a média da pressão intra-ocular na posição supina, nos três grupos, estatisticamente maior que a média da pressão intraocular na posição assentada $(\mathrm{p}<0,001)$.

Houve diferença significativa entre as médias da PIO na posição supina entre os três grupos. O grupo GPAA teve uma média de PIO significativamente maior que os outros dois grupos $(\mathrm{p}<0,001)$, e o grupo com GPN teve uma média significativamente maior que o grupo de $0 \mathrm{~N}$ (Tabela 3 ). A tabela 4 mostra que, na posição assentada, a PIO também foi estatisticamente mai-

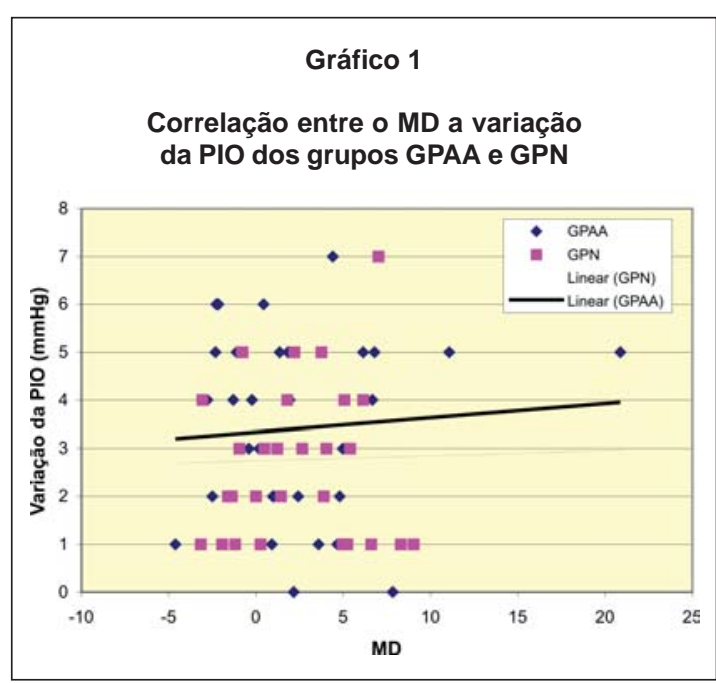

or no grupo GPAA e menor no grupo ON $(\mathrm{p}<0,001)$.

Não houve diferença estatisticamente significativa entre as médias da flutuação da PIO, entre as posições supina e assentada, entre os três grupos $(\mathrm{p}<0,001)$ como mostra a tabela 5 .

A tabela 6 revela que não houve correlação linear entre a flutuação da PIO, entre as posições supina e assentada, e o índice MD da perimetria computadorizada nos grupos GPAA $\left(\mathrm{r}_{\mathrm{s}}=-0,04 ; \mathrm{p}=0,81\right)$ e GPN $\left(\mathrm{r}_{\mathrm{s}}=\right.$ $0,003 ; \mathrm{p}=0,89)$ (Gráfico 1).

Não houve, ainda, diferença estatisticamente significativa da flutuação da PIO entre as posições supina e assentada, entre o melhor olho e o pior olho de cada paciente, de acordo com o índice MD da perimetria computadorizada (Tabela 7).

\section{Discussão}

Foi encontrada diferença significativa entre as medidas da PIO aferidas com o paciente em posição supina e assentada nos três grupos. A PIO foi maior nos pacientes em posição supina. O resultado obtido foi semelhante ao encontrado em trabalhos previamente publicados na literatura ${ }^{1-2,13}$.

Não foi encontrada diferença significativa quando comparada a variação da pressão em posição supina e assentada entre os três grupos. Outro estudo ${ }^{14}$ também não encontrou diferença significativa na variação da PIO relacionada à mudança de postura em pacientes com hipertensão ocular, GPN e ON. No entanto, em outro trabalho que comparou pacientes com GPAA, GPN e voluntários com olhos normais, os autores observaram 
maior flutuação da PIO entre as posições supina e assentada em pacientes com GPN ${ }^{8}$.

A variação da PIO com a mudança de postura tem sido relacionada com o aumento da pressão venosa episcleral. Foi observado em um estudo ${ }^{4}$, um aumento da pressão venosa episcleral relacionada à mudança de postura e o aumento da pressão venosa episcleral era correspondente ao aumento da PIO medida com tonômetro de aplanação. Quando a PIO foi aferida com tonômetro de identação, essa correspondência não foi constatada. Este fato foi relacionado à expulsão do sangue da coróide provocada pela identação, sugerindo que esta tonometria não seria eficaz na avaliação da PIO correlacionada a alterações vasculares. Autores de outro estudo ${ }^{15}$, no entanto, observaram que o aumento da pressão venosa episcleral era maior que o aumento da PIO com a mudança de postura.

Foi observado ainda, um defeito de autoregulação do fluxo sanguíneo da artéria central da retina durante a mudança de posição ${ }^{16}$.

O aumento da pressão venosa episcleral também seria responsável pelo aumento da pressão intra-ocular provocado pela inversão de posição supina para pronoposição $^{14}$, não havendo interferência da regulação do fluxo do humor aquoso ${ }^{17}$.

A detecção de picos de pressão pela manhã com o paciente em posição supina pode ser importante não apenas para o diagnóstico, como também para avaliação clínica de pacientes com glaucoma ${ }^{3,5.7}$. Pacientes com suspeita de glaucoma de pressão normal podem mudar o diagnóstico para glaucoma primário de ângulo aberto, ao serem detectados picos de pressão pela manhã com o paciente em posição supina ${ }^{6-7,18-19}$

Tem sido relatada, em pacientes glaucomatosos, uma maior variação da PIO no olho com maior defeito de campo visual relacionada à mudança de posição?. Outros autores encontraram maior progressão dos defeitos de campo visual em portadores de GPAA, no olho que apresenta maior flutuação da PIO relacionada à mudança postura ${ }^{10}$. No presente estudo não foi encontrada correlação significativa entre a variação da PIO e defeitos de campo visual em pacientes com GPAA e GPN. Os nossos resultados vão de encontro aos trabalhos citados anteriormente, nos quais os pacientes estavam em tratamento de glaucoma. Os resultados deste estudo podem ser explicados pelo fato de que foram incluídos apenas pacientes que ainda não haviam iniciado o tratamento antiglaucomatoso. Este fato representa uma limitação deste estudo para avaliar a relação entre a variação postural da PIO e os defeitos de campo visual em portadores de glaucoma submetidos a tratamento antiglaucomatoso.

Os resultados sugerem que existe um aumento da PIO relacionado à mudança da posição assentada para supina em pacientes com GPAA, GPN e ON. No entanto, não foi encontrada diferença significativa da variação da PIO entre os três grupos e não foi observada correlação significativa dos defeitos de campo visual com a variação da PIO decorrente da mudança e posição.

\section{AbSTRACT}

Purpose: To evaluate intraocular pressure variation due to postural changing of supine to sitting position and, to correlate this variation with visual field defects in primary open angle and normal tension glaucoma patients, as well as normal eyes. Methods: Thirty-two eyes of 32 POAG patients, 30 eyes of 30 NTG patients and 20 eyes of 20 normal patients were evaluated in the study. Intraocular pressure was measured at 60 'clock in supine position and 5 minutes after in sitting position for further correlation between intraocular pressure behavior after postural changing and visual field defects Kruskal-Wallis, Wilcoxon, Mann-Whitney and Spearman linear correlation non-parametric tests were used for statistical analysis. Inherent to the methods used, p-values below 0,05 were considered to ensure statistical relevance. Results:Relevant results were observed between intraocular pressure measured in supine and sitting position in all groups, disclosing statistical significance $(p<0,001)$. However, this statistical relevance was not seen when compared among the 3 groups studied $(p=0,17)$. Moreover, visual field defects, expressed by MD index, did not point to have statistical correlation to intraocular pressure variation after postural changing in POAG $(P=0,81)$ and NTG $(p=0,89)$.Conclusion: The results suggest there is a significant increase in intraocular pressure due to postural changing in primary open angle and normal tension glaucoma patients, as well as in normal eyes. However, the raise in intraocular pressure neither was significant when compared among the 3 groups nor was consistent with visual field defects.

Keywords: Open-angle glaucoma/diagnosis; Intraocular pressure; Diagnostic techniques, ophthalmological; Ocular tonometry/methods; Case control studies

\section{RefERÊNCIAS}

1. Anderson DR, Grant WM. The influence of position on intraocular pressure. Invest Ophthalmol. 1973; 12(3): 204-12.

2. Weber AK, Price J. Pressure differential of intraocular pressure measured between supine and sitting position. Ann Ophthalmol. 1981; 13(3):323-6. 
3. Rodrigues LD, Silva MRBM, Schellini SA, Jorge EN. Picos de pressão intra-ocular: comparação entre curva tensional diária, minicurva e medida da pressão intra-ocular às 6 horas. Arq Bras Oftalmol. 2004; 67(1):127-31.

4. Krieglstein GK, Waller WK, Leydhecker W. The vascular basis of the positional influence of the intraocular pressure. Albrecht Von Graefes Arch Klein Exp Ophthalmol. 1978; 206(2): 99-106.

5. Leonard TJ, Kerr Muir MG, Kirkby GR, Hitchings PA. Ocular hypertension and posture. Br J Ophthalmol. 1983; 67(6):362-6.

6. Meier-Gibbons F, Stürmer J, Gloor B. [Normal pressure glaucoma: a diagnostic challenge]. Klin Monatsbl Augenheilkd. 1995; 206(3):157-60. German.

7. Mardin CY, Jonas J, Michelson G, Jünemann A. [Are there genuine and pseudo-normal pressure glaucomas? Body position-dependent intraocular pressure values in normal pressure glaucoma]. Klin Monatsbl Augenheilkd. 1997; 211(4):235-40. German.

8. Tsukahara S, Sasaki T. Postural change of IOP in normal persons and in patients with primary wide open-angle glaucoma and low-tension glaucoma. Br J Ophthalmol. 1984; 68(6):389-92.

9. Hirooka K, Shiraga F. Relationship between postural change of the intraocular pressure and visual field loss in primary open-angle glaucoma. J Glaucoma. 2003; 12(4):379-82.

10. Kiuchi T, Motoyama Y, Oshika T. Relationship of progression of visual field damage to postural changes in intraocular pressure in patients with normal-tension glaucoma. Ophthalmology. 2006; 113(12): 2150-5. Comment in: Ophthalmology. 2007; 114(12):2362; author reply 2362. Ophthalmology. 2007; 114(7):1413; author reply 1413-4.

11. Jonas JB, Budde WM, Panda-Jonas S. Ophthalmoscopic evaluation of the optic nerve head. Surv Ophthalmol. 1999; 43(4):293-320. Review.
12. Comparison of glaucomatous progression between untreated patients with normal-tension glaucoma and patients with therapeutically reduced intraocular pressures. Collaborative Normal-Tension Glaucoma Study Group. Am J Ophthalmol. 1998; 126(4):487-97. Erratum in: Am J Ophthalmol. 1999;127(1):120.

13. Liu JH, Zhang X, Kripke DF, Weinreb RN. Twenty-four-hour intraocular pressure pattern associated with early glaucomatous changes. Invest Ophthalmol Vis Sci. 2003; 44(4): 1586-90.

14. Yamabayashi S, Aguilar RN, Hosoda M, Tsukahara S. Postural change of intraocular and blood pressures in ocular hypertension and low tension glaucoma. Br J Ophthalmol. 1991; 75(11): 652-5. Comment in: Br J Ophthalmol. 1991; 75(11):643.

15. Kaskel D, Müller-Breitenkamp R, Wilmans I, Rudolf H, Jessen $\mathrm{K}$. [The influence of changes in body position on intraocular pressure, episcleral venous pressure, and blood pressure (author's transl)]. Albrecht Von Graefes Arch Klin Exp Ophthalmol. 1978; 208(4):217-28. German.

16. Friberg TR, Sanborn G, Weinreb RN. Intraocular and episcleral venous pressure increase during inverted posture. Am J Ophthalmol. 1987; 103(4):523-6

17. Carlson KH, McLaren JW, Topper JE, Brubaker RF. Effect of body position on intraocular pressure and aqueous flow. Invest Ophthalmol Vis Sci. 1987; 28(8): 1346-52.

18. Calixto N, Meira DM, Cronemberger S. Estudo de pacientes com suspeita diagnóstica de glaucoma de pressão normal. Rev Bras Oftalmol. 1997; 56(11): 823-34.

19. Meirelles SHS, Stohler NSF, Mariano MP, Yamane R, Yamane Y. Espessura corneana no glaucoma de pressão normal. Rev Bras Oftalmol. 2001; 60(1):56-64. 